\title{
Familial multiple sclerosis with repetitive relapses of manic psychosis in two patients (mother and daughter) ${ }^{1}$
}

\author{
Pedro J. Modrego ${ }^{\mathrm{a}, *}$ and Jaime Ferrández ${ }^{\mathrm{b}}$ \\ ${ }^{a}$ Neurology Unit, Hospital del Insalud de Alcañiz. \\ 44600, Alcañiz, Spain \\ ${ }^{\mathrm{b}}$ Mental Health Unit, Insalud de Alcañiz 44600, \\ Alcañiz, Spain
}

The clinical presentation of multiple sclerosis (MS) with psychiatric symptoms is uncommon but it is believed that MS patients are twice as likely to be afflicted with bipolar disorder as the general population. We report two cases (mother and daughter) of MS presenting with bipolar disorder in the form of recurrent manic psychosis and whose outcome was favourable with neuroleptics and corticosteroids. In both cases we found multiple hypersignal lesions on brain magnetic resonance imaging (MRI), especially in the right frontal lobe where we observed signs of activity. Apart from clinical and radiological concordance, the patients exhibited similar class I HLA alleles and identical class II HLA alleles. We focused discussion on whether there may be a common genetic susceptibility to both illnesses or whether MS caused psychiatric manifestations. The coincidence of psychiatric and neurological symptoms in most relapses supports the second hypothesis.

Keywords: Multiple sclerosis, bipolar disorder, manic psychosis

\section{Introduction}

Emotional disturbances are highly prevalent in patients with multiple sclerosis (MS) but little attention has been paid to this domain of health. By affective or mood disorders are meant abnormal emotional states such as depression, anxiety, pathological weeping or

\footnotetext{
${ }^{1}$ This paper was presented at the tenth meeting of the European Neurological Society in Jerusalem, June 2000.

* Corresponding author: Dr. P.J. Modrego, Avda Juan Carlos I, 21, 50009 Zaragoza, Spain. Fax: +34 97883097.
}

laughing, euphoria or mania that are accompanied by disturbances of sleep and appetite as well as by characteristic patterns of thought and behaviour [10]. The frequency of affective bipolar disorder in MS patients was twice that reported for the general population in Monroe County, New York [15]. Another previous report showed that 13 out of 100 consecutive MS patients had bipolar disorder versus $1 \%$ in the general population [7]. Only preliminary reports point to familial clustering of MS and bipolar disorder [3,16] but this has to be confirmed.

We report two familial cases (mother and daughter) of MS presenting with repetitive relapses of bipolar disorder (mania) in the context of active MS.

\section{Patients and methods}

Both patients fulfilled the criteria of Poser et al for clinically definite MS [14] as well as the modified criteria of Goodkin et al. for multiply affected families [5]. The diagnosis of bipolar disorder was made by means of structured interview in accordance with the criteria of DSM-IV [1].

Laboratory tests including HLA typing and conventional spin echo Gadolinium-enhanced-Magnetic Resonance Imaging (MRI) were performed on both patients.

\subsection{Observations}

Case 1. A 42 year old woman, whose father committed suicide without evident previous history of psychiatric illnesses, was well until December, 1988 when she began with sleep disturbances, hyperactivity on homework and bulimia, aggressive and accusatory speech, racing and delirant thought with religious content. She spent much money in Christmas presents and the mood was variable alternating irritability with euphoria. Ini- 
tially she was treated with haloperidol, $6 \mathrm{mg}$ per day without significant improvement.

In January , 1989 she was admitted to the hospital and treated with higher doses of haloperidol and lithium carbonate with remarkable improvement in her mental status. An autoimmune hypothyroidism was detected by means of specific blood tests and treated with levothroid. The patient was discharged with the diagnosis of manic-depressive psychosis and continued taking lithium salts.

She was asymptomatic until August 1995 when complained of severe paraesthesias in both arms, restlessness, excitation, suspiciousness, and early awakening. Her husband observed mood variability, visual hallucinations and verbosity. Haloperidol and levopromazine were added to treatment with rapid improvement in several days. One month after, the neuroleptics were withdrawn and the patient continued with lithium salts.

In June, 1999 she was seen again by a psychiatrist because of recurrence of symptoms: verbal aggressiveness, accusatory sentences, flight of ideas, distractibility, irritability, insomnia, and visual hallucinations. Then she was diagnosed of bipolar disorder and treated again with haloperidol and levopromazine without improvement on that occasion. Both drugs were changed for trifluoperazine and risperidone under which she developed severe akinesia and rigidity, excessive drooling and inability to walk. A brain computed tomography revealed remarkable atrophy. Risperidone and trifluoperazine were withdrawn and changed for haloperidol and levopromazine again. Once extrapyramidal side-effects disappeared, a magnetic resonance (MRI) scan of the brain was performed. T2-weighted gadolinium enhanced images indicated multiple hypersignal lesions in periventricular white matter, cerebellum, corpus callosum and a large and active lesion on right frontal lobe with peripheral oedema (see Fig. 1). On clinical examination she exhibited ataxic gait without paresis. Standard blood tests revealed no abnormalities, the erythrocyte sedimentation rate was normal, tests for autoantibodies (antinuclear, anti-DNA, anti-SS-A, antiSS-B, anti-thyroglobulin) were negative, antimicrosomal antibodies were positive (titre:1/320) in relation to dysimmune hypothyroidism previously mentioned. Serologic tests for Lyme disease were also negative. Taking into account the clinical and radiological data the diagnosis of clinically definite multiple sclerosis was made and the patient was treated with high doses of methyl prednisolone plus tapering doses of prednisone. The outcome was favourable and she continues taking low doses of levopromazine and lithium carbonate. Her current mental status shows no abnormalities but mild ataxia remains on clinical examination.

In October, 2000 the patient presented with another bout, on this occasion with paraparesis and expansive mood in the form of hypomania. Once again corticosteroids produced remarkable improvement of her symptoms but the gait remains somewhat spastic and ataxic. The HLA haplotypes are reported in Table 1.

Case 2. A 19 year old woman, who was the daughter of case 1, was seen firstly in May, 1995 because bilateral manual clumsiness. She did not refer any previous illness. The neurological examination only revealed maladroitness of both hands without distal weakness. A MRI study of the brain revealed hypersignal lesions in periventricular areas and especially in the right frontal lobe (see Fig. 2), compatible with demyelinating disease. Two months after the first consultation she began with flight of ideas, disinhibition, expansive mood, verbosity, restlessness, irritability, wakefulness and deliriant thinking. She was evaluated by a psychiatrist and diagnosed as bipolar disorder related to probable multiple sclerosis. Under treatment with neuroleptics and corticosteroids the outcome was favourable and the symptoms remitted almost completely in two months.

In May, 1996 she was admitted to the hospital because of weakness of the legs, urinary incontinence and agitation. The clinical examination revealed paraparesis with bilateral extensor plantar responses. On mental examination the patient was disinhibited with socially intrusive demeanour. She exhibited deliriant thought (“... my mother has the devil inside ...", she said), excessive verbosity, and visual hallucinations. Temperature and blood pressure were normal. The standard blood tests, autoantibodies tests, and Lyme disease serologic tests were performed without finding abnormalities. A lumbar puncture disclosed a clear CSF without cells, with normal biochemical composition; the proteinogram did not reveal abnormalities, the IgG index was 0.45. A diagnosis of bipolar disorder (manic psychosis) was made again in the context of a new relapse of MS. The treatment with high doses of metilprednisolone plus neuroleptics resulted in progressive improvement of mental and motor functions. One month after, she only had mild unsteadiness of gait. The mood and behaviour returned to normal and, up to date, she has not had more relapses. However her academic performance has declined, especially in memory tasks, and she has had to leave her studies at high school. At the present time she is working and is not under any treatment.

The HLA haplotypes are reported in Table 1. The class II HLA alleles are identical to those obtained in her mother. 


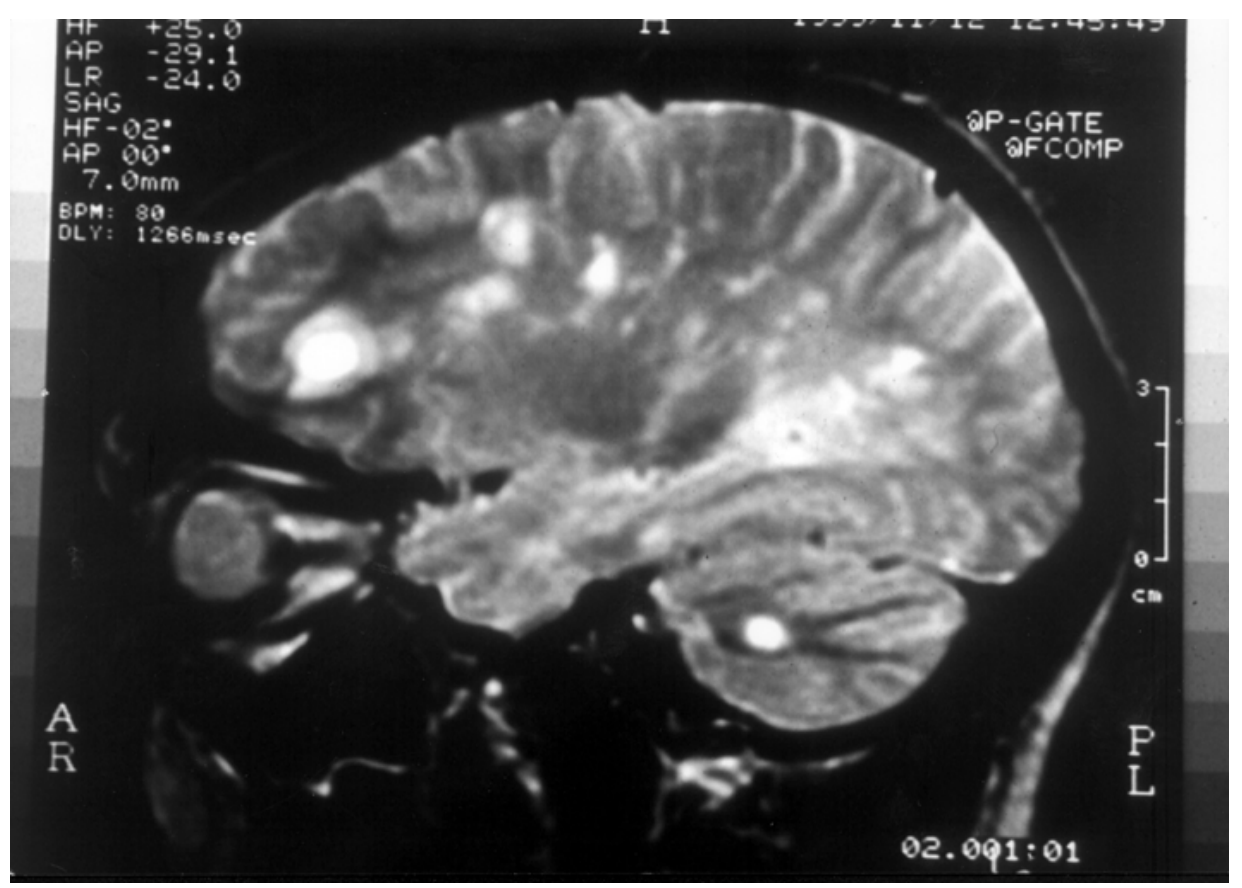

Fig. 1. T2-weighted gadolinium-enhanced MRI showing hyperintense lesions in the corpus callosum, cerebellum, periventricular white matter, and right frontal lobe. The presence of oedema in the frontal lesion suggest that it is active.

Table 1

Age, MRI findings, and HLA alleles

\begin{tabular}{|c|c|c|}
\hline Variables & Mother & Daughter \\
\hline Current age & 42 & 19 \\
\hline Age at onset & 30 & 15 \\
\hline Date of diagnosis & September, 1999 July, 1995 & \\
\hline Findings on MRI & $\begin{array}{l}\text { Hypersignal lesions on periventricular } \\
\text { white matter, Corpus callosum, Cerebel- } \\
\text { lum, right frontal lobe }\end{array}$ & $\begin{array}{l}\text { Hypersignal lesions periventricular white } \\
\text { matter, right frontal lobe }\end{array}$ \\
\hline HLA-Alleles A & $\mathrm{A} 1, \mathrm{~A} 30$ & A1, A32 \\
\hline HLA-Alleles B & B8, B51, BW4, BW6 & B8, B44, BW4, BW6 \\
\hline HLA-Alleles DR & DRB1*03(17), DRB1*04, DRB3, DRD4 & DRB1*03(17), DRB1*04, DRB3, DRD4 \\
\hline HLA-Alleles DQ & DQB1*02, DQB1*03(8) & DQB1*02, DQB1*03(8) \\
\hline
\end{tabular}

\subsection{Discussion}

MS can manifest as neuropsychiatric disturbances even in the absence of physical disabilities. However a small proportion of all psychiatric admissions are due to MS. Only ten MS patients among a total of 2720 admissions in three psychiatric units were reported to have a history of mania and manic psychosis [13]. Bipolar disorder and multiple sclerosis are clinically cyclical diseases in nature and both may show familial occurrence. Among $10 \mathrm{MS}$ patients with bipolar disorder, Schiffer et al. [15] found that 5 patients had first or second-degree relatives with affective disorders. In another study with 56 MS patients, 3 had family history of MS and bipolar disorder but no concordance between mother and daughter was reported in any of the patients [16].

It is not clear whether bipolar disorder was or was not an initial manifestation of MS in the first case because in the first relapse the patient did not undergo an appropriate neurological examination. In the remaining three bouts she also showed neurological symptoms. In the case 2 mania was accompanied by other neurological symptoms in both relapses and both types of symptoms improved with treatment at the same time. In the first bout, the mother only presented with psychiatric symptoms but the remarkable atrophy of the brain and the presence of old lesions on MRI suggest that the disease has started a long time before. The same hypothesis was claimed in a report of seven MS patients who 


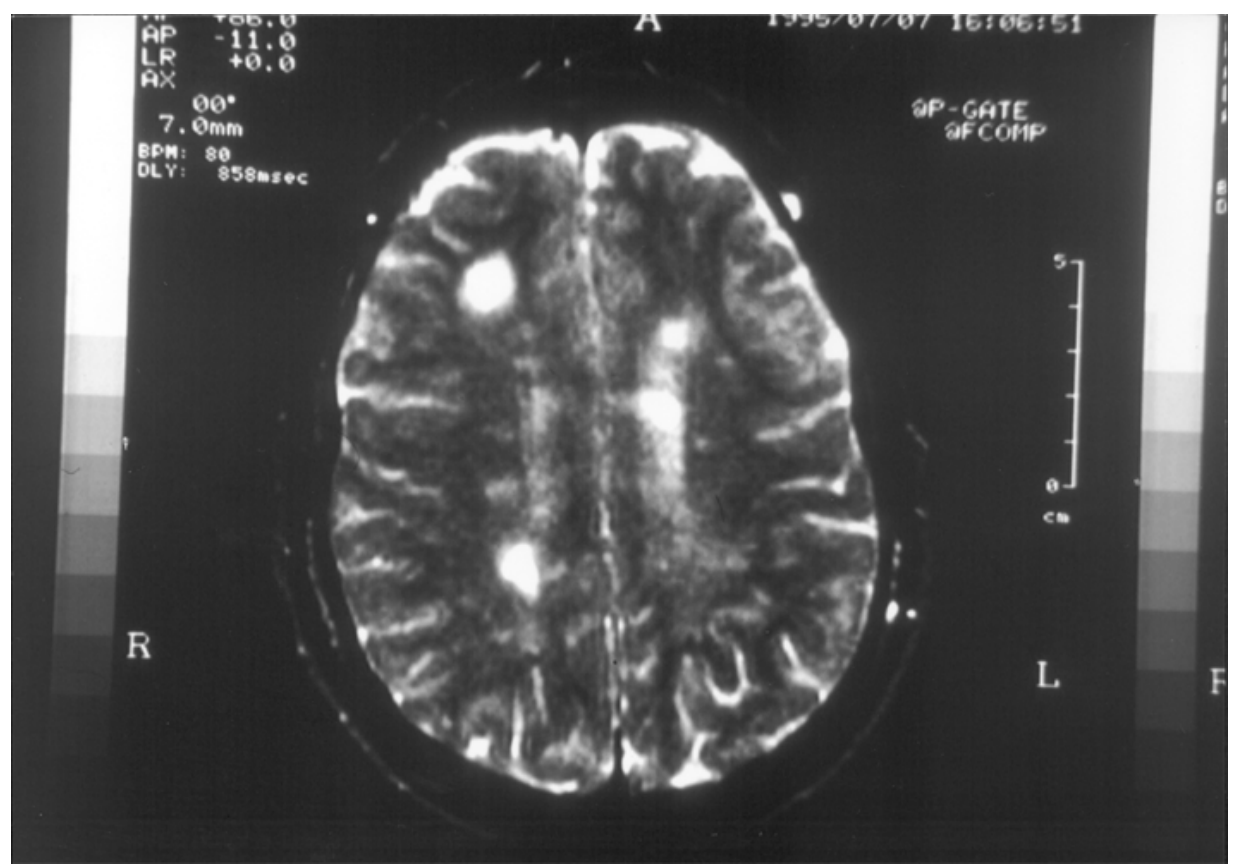

Fig. 2. T2-weighted Gadolinium-enhanced-MRI of the brain. Hypersignal lesions in periventricular white matter and a large lesion in the right frontal lobe.

had a preceding history of bipolar disorder years before any neurological symptom [6]. However we also acknowledge there may be two arguments weakening the hypothesis that MS caused bipolar disorder: the chance of having identical class II HLA alleles in this mother/daughter pair is four regardless of concordance for the disease, and the fact of suicide in a member of the family.

Previous reports in Spain point to the link between MS and HLA DR2. In Madrid this allele was found in $47 \%$ of $121 \mathrm{MS}$ patients and in $24 \%$ of controls [4] but this relationship was not strong enough in Asturias [8]. In our region we dispose of the data obtained in 36 patients and 895 unrelated controls [12]; DR2 antigen was detected in $39 \%$ of patients and in $19 \%$ of controls. All patients positive for DR2 antigen showed the DRB1 1501 and DRB5 0101 alleles. In the DQ class antigens no differences were observed as DQ1 antigen was observed in $29 \%$ of patients versus $31 \%$ of controls. Numerous articles about the association between HLA and affective disorders have been published but the results are largely inconclusive. Although non random transmission of HLA haplotypes was observed in a large canadian study, however the role of HLA genes has to be considered in the spectrum of a multifactorial etiology of affective disorders [17]. A small study conducted in Spain showed a weak association between B29 and B21 haplotypes and bipolar disorder [18].
The topographic correlates of mania have not been completely resolved in MS-related bipolar disorder. Some studies did not report neuroimaging data $[7,15$, 16]. Among the seven cases of Hutchinson et al., MRI was performed on five in whom no common pattern of lesion distribution was observed except for periventricular location in four of them [6]. An autopsy of an elderly woman with postmenopausal onset of bipolar disorder disclosed widespread periventricular lesions [2], however this is a common finding in MS. Demyelination of prefrontal-subcortical circuits has been thought to be responsible for neuropsychiatric symptoms in MS [9]. We hypothesised that the right frontal lesions could be responsible for bipolar disorder in our patients because of their large size and activity signs on MRI. Notwithstanding, we are aware that any lesion that produces disconnection of subcortical circuits may cause mania.

We would raise the importance of treatment of mania in the context of MS with steroids and not treating this problem as purely psychiatric disorder. Some clinicians are concerned about treating manic disorders with steroids, but our reports clearly indicate that the treatment may be helpful and not deleterious when mania occurs in the setting of active MS. It is also worth mentioning that the concordance between mother and daughter for MS only occurs in 3\% of multiplex families [11]. 
In conclusion, the concordance observed in these two familial cases of MS with respect to manic psychosis, the MRI evidence of demyelinating lesions and HLA alleles contributes to a better understanding of MSrelated bipolar disorder.

\section{References}

[1] American Psychiatric Association, Diagnostical and Statistical Manual of Mental Disorders: DSM-IV, Washington, DC, 1994.

[2] M.F. Casanova, M. Kruesi and G. Mannheim, Multiple sclerosis and bipolar disorder: a case report with autopsy findings, J Neuropsychiatry Clin Neurosci 8 (1996), 206-208.

[3] C.L. Cazzullo, E. Smeraldi, M. Gasperini and D. Caputo, Preliminary correlation between primary affective disorders and multiple sclerosis, in: New trends in multiple sclerosis research, C.L. Cazzullo, D. Caputo and A. Ghezzi, eds, Masson Publishing USA Inc, New York, NY, 1983, pp. 57-62.

[4] N. Clerici, M. Hernández, M. Fernández, J. Rosique and J. Alvarez Cermeño, Multiple sclerosis is associated with HLADR2 antigen in Spain, Tissue Antigens 34 (1989), 309-311.

[5] D. Goodkin, T.H. Doolittle and S.S. Hauser et al., Diagnostic criteria for multiple sclerosis research involving multiply affected families, Arch Neurol 48 (1991), 805-807.

[6] M. Hutchinson, J. Stack and P. Buckley, Bipolar affective disorder prior to the onset of multiple sclerosis, Acta Neurol Scand 88 (1993), 388-393.

[7] R.T. Joffe, G.P. Lippert, J.A. Gray, G. Sawa and Z. Horvath, Mood disorders and multiple sclerosis, Arch Neurol 44 (1987), 376-378.

[8] C. López Larrea, D.F. Uría and E. Coto, HLA antigens in multiple sclerosis of northern spanish population, J Neurol Neurosurg Psychiatry 52 (1989), 434-435.

[9] M.F. Mendez, The neuropsychiatry of multiple sclerosis, Int J Psychiatry Med 25 (1995), 123-130.

[10] S.L. Minden and R.B. Schiffer, Affective disorders in Multiple Sclerosis. Review and recommendations for clinical research, Arch Neurol 47 (1990), 98-104.

[11] Multiple Sclerosis Genetics Group, Clinical demographics of multiplex families with multiple sclerosis, Ann Neurol $\mathbf{4 3}$ (1998), 530-534.

[12] M.A. Pina, J.R. Ara, P. Lasierra, P.J. Modrego and L. Larrad, Study of HLA as a predisposing factor and its possible influence on the outcome of multiple sclerosis in the sanitary district of Calatayud, Northern Spain, Neuroepidemiology 18 (1999), 203-209.

[13] D.S. Pine, C.J. Douglas, E. Charles, M. Davis and D. Kahn, Patients with multiple sclerosis presenting to psychiatric hospitals, J Clin Psychiatry 56 (1995), 297-306.

[14] C.M. Poser, D. Paty and L. Scheinberg et al., New diagnostic criteria for multiple sclerosis. Guidelines for research protocols, Ann Neurol 13 (1983), 227-231.

[15] R.B. Schiffer, N.M. Wineman and L.R. Weitkamp, Association between bipolar affective disorder and multiple sclerosis, Am J Psychiatry 143 (1986), 94-95.

[16] R.B. Schiffer, L.R. Weitkamp, N.M. Wineman and S. Guttornsem, Multiple sclerosis and affective disorder: family history, sex, and HLA-DR antigens, Arch Neurol 45 (1988), 1345-1348.

[17] H.C. Stancer, L.R. Weitkamp and E. Persad et al., Confirmation of the relationship of HLA genes to depression and manic depression. II. The Ontario follow-up and analysis of 117 kindreds, Ann Hum Genet 52 (1988), 279-298.

[18] T. Ventura, A. Lobo and J.C. Marco, HLA antigens in bipolar affective disorders, Actas Luso Esp Neurol Psiquiatr Cienc Afines 18 (1990), 339-343. 


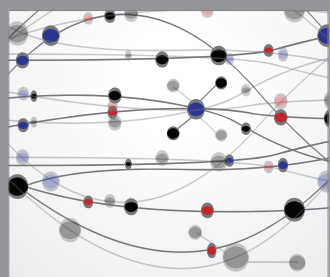

The Scientific World Journal
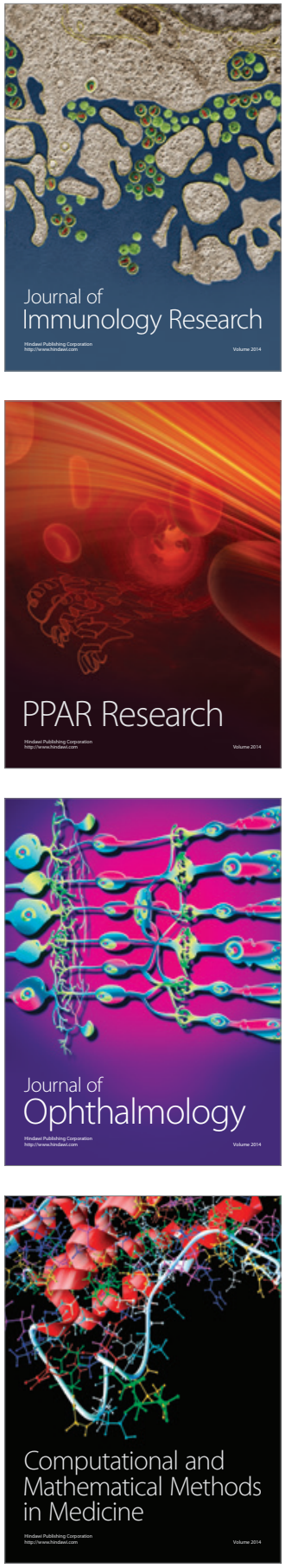

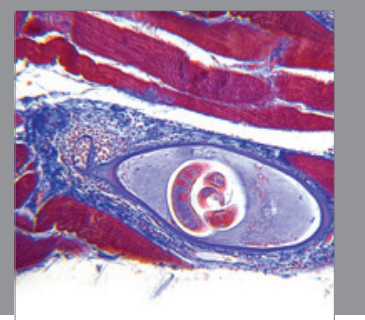

Gastroenterology

Research and Practice
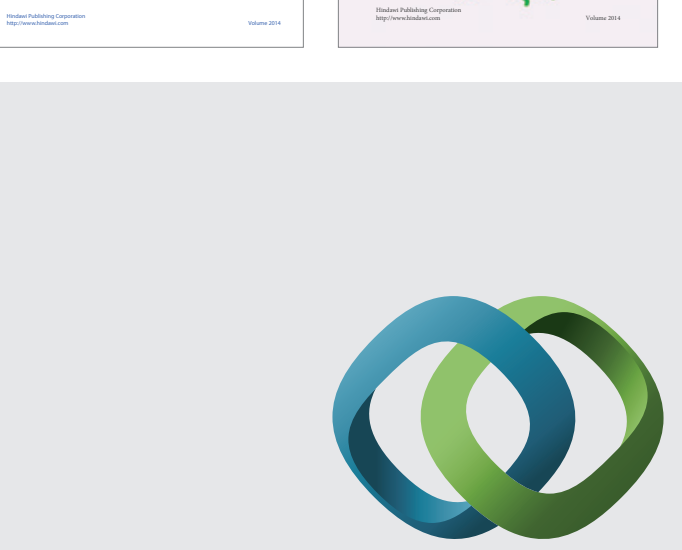

\section{Hindawi}

Submit your manuscripts at

http://www.hindawi.com
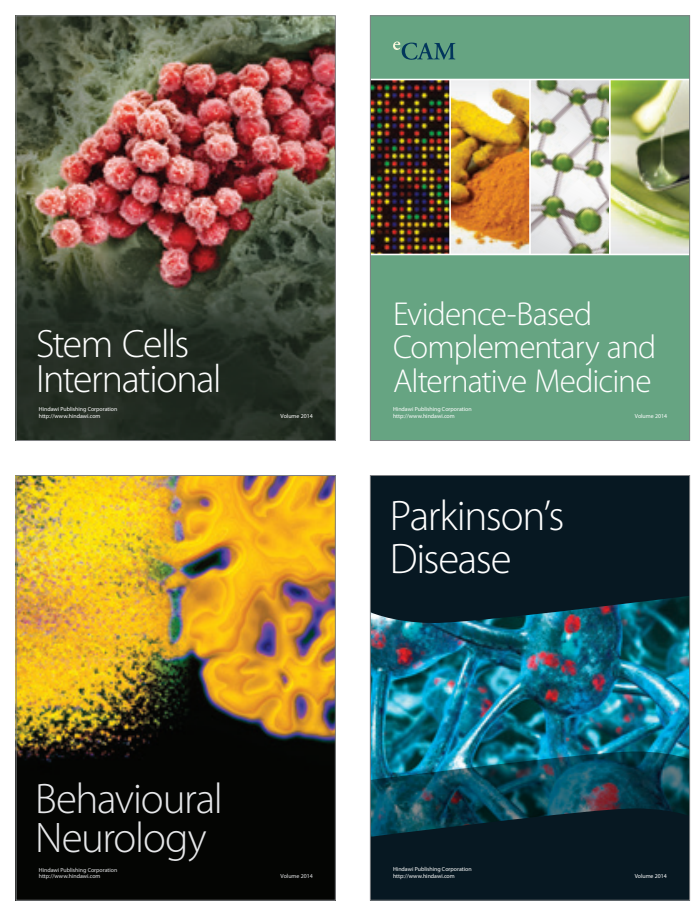

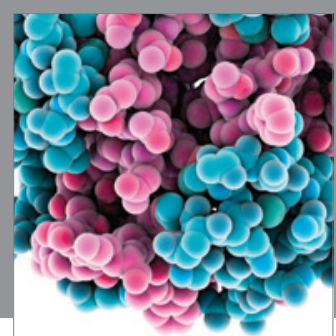

Journal of
Diabetes Research

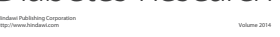

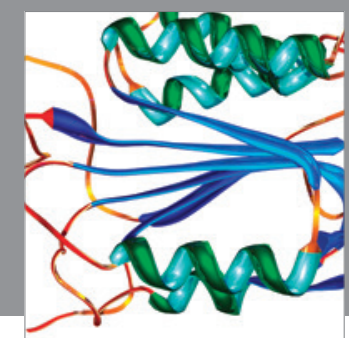

Disease Markers
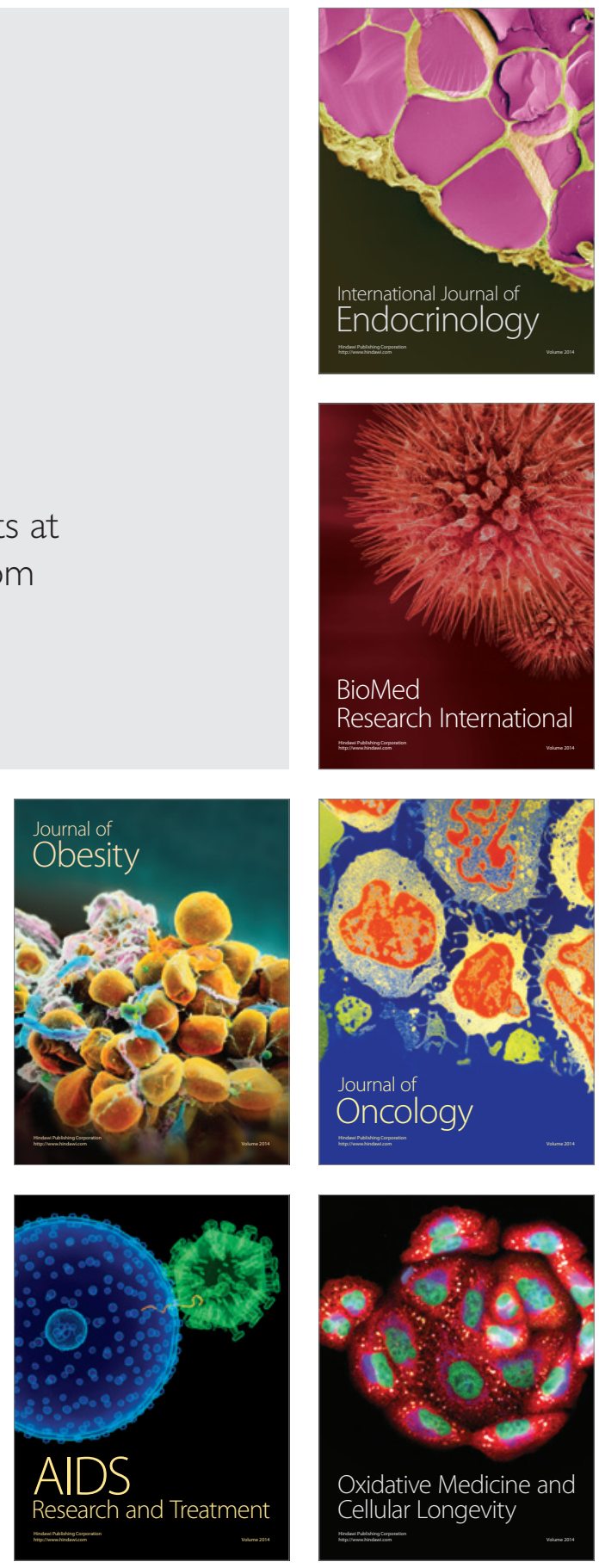